Interactive comment on "The Open-source Data Inventory for Anthropogenic Carbon dioxide $\left(\mathrm{CO}_{2}\right)$, version 2016 (ODIAC2016): A global, monthly fossil-fuel $\mathrm{CO}_{2}$ gridded emission data product for tracer transport simulations and surface flux inversions" by Tomohiro Oda et al.

Tomohiro Oda et al.

tomohiro.oda@nasa.gov

Received and published: 20 November 2017

Dear Anonymous reviewer \#2

Thank you for your comments and suggestions. Our response will be submitted as a supplement PDF file.

Sincerely, 


\section{Tomohiro Oda}

ESSDD

Please also note the supplement to this comment:

https://www.earth-syst-sci-data-discuss.net/essd-2017-76/essd-2017-76-AC1-

supplement.pdf

Interactive comment on Earth Syst. Sci. Data Discuss., https://doi.org/10.5194/essd-2017-76, 2017.

Interactive

comment 